\title{
Branch retinal artery occlusion following radiation therapy to the head and neck: a case report
}

\author{
Helen Jiang ${ }^{1}$, Maxwell S Stem ${ }^{2}$ and Jerome I Finkelstein ${ }^{2 *}$
}

\begin{abstract}
Background: Previous studies have established that radiation to the head and neck leads to atherosclerosis and stenosis of the carotid artery and subsequent increased stroke risk, but the ophthalmic sequella following cervical irradiation is less well-defined.

Case presentation: We present a single case of branch retinal artery occlusion (BRAO) in a 55 year-old Caucasian male seen at the University of Michigan in 2008 following unilateral head and neck radiation.

Conclusion: This case demonstrates that patients receiving radiation to the head and neck may be at increased risk for developing a BRAO secondary to atherosclerotic changes of vessels adjacent to the radiation target. Given this risk, it may be reasonable to obtain carotid artery imaging in patients with a history of cervical radiation who present with sudden or transient visual field defects, even in the absence of other conventional risk factors for atherosclerosis.
\end{abstract}

Keywords: Radiation, Head and neck, Atherosclerosis, Branch retinal artery occlusion (BRAO)

\section{Background}

Prior studies have demonstrated that radiation therapy to the head and neck leads to carotid artery stenosis [1]. Furthermore, head and neck radiation increases stroke risk [2]. However, the relationship between radiationinduced carotid artery damage and embolic ophthalmic events has been less well explored. We present a case of branch retinal artery occlusion (BRAO) in a patient without known cardiovascular disease following unilateral head and neck radiation.

\section{Case presentation}

A 55-year-old Caucasian man with a history of squamous cell carcinoma of the left tonsil treated with neck dissection, chemotherapy, and radiation therapy eight years previously presented to a comprehensive ophthalmologist complaining of a gray spot in the upper right quadrant of his left eye that began four days ago. He denied any pain, flashes, or floaters. Past medical history was significant for hyperlipidemia, and family history was significant for coronary artery disease (CAD).

\footnotetext{
*Correspondence: jifink@umich.edu

${ }^{2}$ Department of Ophthalmology and Visual Sciences, University of Michigan Medical School, Ann Arbor, USA

Full list of author information is available at the end of the article
}

Examination of the left eye revealed a vision of 20/20, intraocular pressure of $16 \mathrm{mmHg}$, and a normal slit lamp exam (SLE). Dilated fundus exam (DFE) of the left eye demonstrated a region of retinal edema along the inferotemporal arcade extending just below the fovea. Multiple Hollenhorst plaques were noted throughout the same area, consistent with a diagnosis of BRAO. The fundus photograph and fluorescein angiogram from the day after presentation are shown in Figure 1. Examination of the right eye was normal.

The patient underwent carotid artery ultrasound, which revealed a fully patent right carotid but evidence of a heterogeneous plaque measuring 70-99\% diameter stenosis in the left carotid. A transthoracic echocardiogram did not reveal any intracardiac sources of emboli. With his BRAO and significant ipsilateral carotid artery stenosis, the patient met clinical criteria for undergoing a carotid endarterectomy [3] and was referred to a vascular surgeon for this definitive treatment.

Three months after the patient's endarterectomy, he was noted to have $20 / 20$ vision in the left eye with residual visual field defect in the superonasal quadrant of his left eye, corresponding to the original area of retinal nonperfusion (Figure 2).

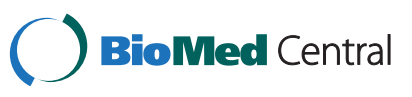




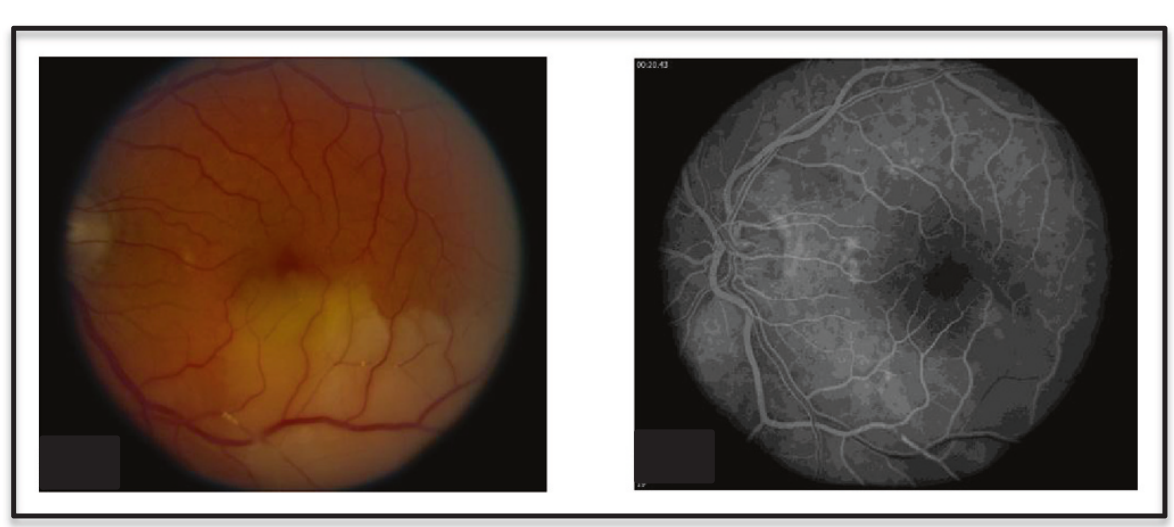

Figure 1 The fundus photograph demonstrates multiple Hollenhurst plaques and an area of retinal edema in the left eye. The fluoroscein angiogram highlights inferior vascular filling defects.

\section{Conclusions}

Radiation therapy promotes the development of atherosclerosis by damaging the vascular endothelium [1]. Radiation-induced vascular damage may occur through several potential mechanisms such as increased proteoglycan deposition and/or inflammatory cell infiltration [4].

Our patient presented with a BRAO of the left eye eight years after radiation therapy to the head and neck. The radiation-induced damage to the left carotid artery likely precipitated the BRAO. Arguably, the patient had several risk factors for conventional atherosclerosis including hyperlipidemia and a family history of CAD. However, the presence of significant stenosis in the carotid artery ipsilateral to the site of irradiation, the absence of atherosclerotic disease in the contralateral carotid, and the lack of atherosclerotic retinal vessels on DFE all support the hypothesis that radiation was the primary factor responsible for the patient's left carotid artery stenosis. The ophthalmic literature contains one case of central retinal artery occlusion (CRAO) following neck irradiation [5]. However, that patient developed a CRAO contralateral to the site of irradiation and was found to have bilateral carotid stenosis on subsequent imaging. To the best of our knowledge, a case of unilateral radiation-induced atherosclerosis leading to $\mathrm{BRAO}$ has not been reported previously in the literature.

This case reiterates that patients who have received head and neck radiation may be at increased risk for the development of atherosclerosis in the arteries proximal to the radiation target. While this patient clearly had a BRAO based on fundoscopic exam, it is feasible that such patients could present with transient visual field defects or vision loss without any associated Hollenhorst plaques. Therefore, it may be prudent to obtain carotid artery imaging in patients with a history of head and neck radiation who present with sudden, transient, or prolonged visual field defects, even if the patients have few other risk factors for atherosclerosis or lack pathognomonic Hollenhorst plaques during fundoscopic examination.

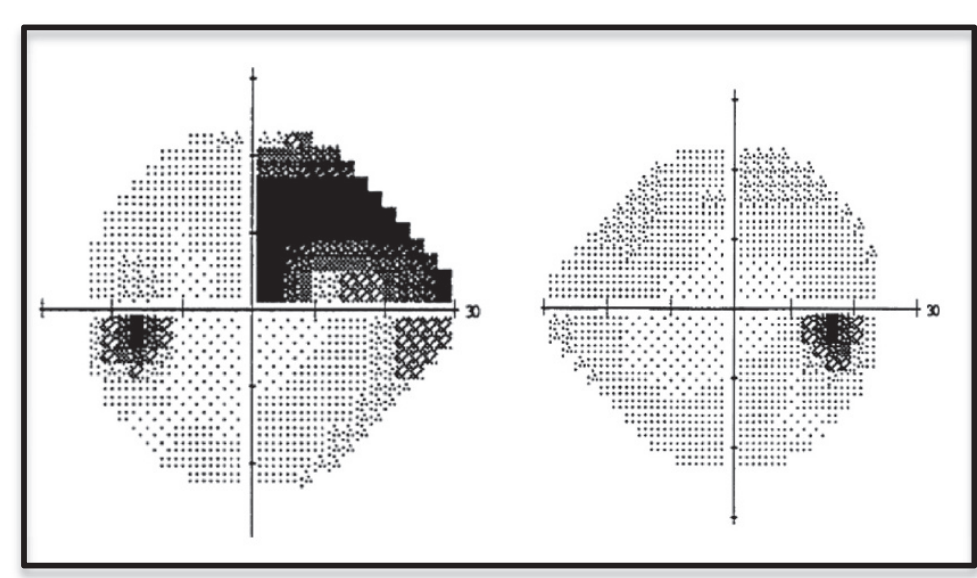

Figure 2 Three months after presentation, the patient had a residual superonasal visual field defect in the left eye. 


\section{Consent}

Written informed consent was obtained from the patient for publication of this Case report and any accompanying images. A copy of the written consent is available for review by the Editor of this journal.

\section{Competing interests}

The authors declare that they have no competing interests.

\section{Authors' contributions}

MS and $\mathrm{HJ}$ performed a background search of the literature and drafted the manuscript. JF provided care for the patient, conceived of this case report, and helped to edit the manuscript. All authors read and approved the final manuscript.

\section{Acknowledgements}

This work was supported in part by the American Diabetes Association Merck Clinical/Translational Postdoctoral Fellowship Award (MSS).

The sponsor or funding organization had no role in the design or conduct of this research.

\section{Author details}

${ }^{1}$ University of Michigan Medical School, Ann Arbor, USA. ${ }^{2}$ Department of Ophthalmology and Visual Sciences, University of Michigan Medical School, Ann Arbor, USA.

Received: 25 August 2013 Accepted: 23 October 2013

Published: 1 November 2013

\section{References}

1. Cheng SW, Wu LL, Ting AC, Lau H, Lam LK, Wei WI: Irradiation-induced extracranial carotid stenosis in patients with head and neck malignancies. Am J Surg 1999, 178(4):323-328.

2. Haynes JC, Machtay M, Weber RS, Weinstein GS, Chalian AA, Rosenthal DI: Relative risk of stroke in head and neck carcinoma patients treated with external cervical irradiation. Laryngoscope 2002, 112(10):1883-1887.

3. North American Symptomatic Carotid Endarterectomy Trial Collaborators: Beneficial effect of carotid endarterectomy in symptomatic patients with high-grade carotid stenosis. N Engl J Med 1991, 325(7):445-453.

4. Russell NS, Hoving S, Heeneman S, et al: Novel insights into pathological changes in muscular arteries of radiotherapy patients. Radiother Oncol 2009, 92(3):477-483.

5. Evans $L S$, Van de Graaff WB, Baker WH, Trimble SN: Central retinal artery occlusion after neck irradiation. Am J Ophthalmol 1992, 114(2):224-225.

doi:10.1186/1471-2415-13-66

Cite this article as: Jiang et al: Branch retinal artery occlusion following radiation therapy to the head and neck: a case report. $B M C$

Ophthalmology 2013 13:66.

\section{Submit your next manuscript to BioMed Central and take full advantage of:}

- Convenient online submission

- Thorough peer review

- No space constraints or color figure charges

- Immediate publication on acceptance

- Inclusion in PubMed, CAS, Scopus and Google Scholar

- Research which is freely available for redistribution 